\title{
Soft X-ray imaging of thick carbon-based materials using the normal incidence multilayer optics
}

\author{
I.A. Artyukov ${ }^{\mathrm{a}, *}$, R.M. Feschenko ${ }^{\mathrm{a}}$, A.V. Vinogradov ${ }^{\mathrm{a}}$, Ye.A. Bugayev ${ }^{\mathrm{b}}$, O.Y. Devizenko ${ }^{\mathrm{b}}$, \\ V.V. Kondratenko ${ }^{\text {b }}$, Yu.S. Kasyanov ${ }^{c}$, T. Hatano ${ }^{d}$, M. Yamamoto ${ }^{d}$, S.V. Saveliev ${ }^{\text {e }}$ \\ a P.N.Lebedev Physical Institute, 53 Leninsky Prospekt, Moscow 119991, Russia \\ b National Technical University “Kharkov Polytechnic Institute”, 21 Frunze St., Kharkov 61002, Ukraine \\ c A.M.Prokhorov General Physics Institute, 38 Vavilov St., Moscow 119991, Russia \\ d Institute of Multidisciplinary Research for Advanced Materials, Tohoku University, 2-1-1 Katahira, Aoba-ku, Sendai 980-8577, Japan \\ e Institute of Human Morphology, 3 Tsourupa Str., Moscow 117418, Russia
}

\section{A R T I C L E I N F O}

\section{Article history:}

Received 28 January 2010

Received in revised form 21 June 2010

Accepted 21 June 2010

\section{Keywords:}

Soft X-ray microscopy

Carbon window

$\mathrm{X}$-ray multilayer mirror

Laser plasma

\begin{abstract}
A B S T R A C T
The high transparency of carbon-containing materials in the spectral region of "carbon window" $(\lambda \sim 4.5-5 \mathrm{~nm})$ introduces new opportunities for various soft X-ray microscopy applications. The development of efficient multilayer coated X-ray optics operating at the wavelengths of about $4.5 \mathrm{~nm}$ has stimulated a series of our imaging experiments to study thick biological and synthetic objects. Our experimental set-up consisted of a laser plasma X-ray source generated with the 2nd harmonics of $\mathrm{Nd}$-glass laser, scandium-based thin-film filters, Co/C multilayer mirror and X-ray film UF-4. All soft $\mathrm{X}$-ray images were produced with a single nanosecond exposure and demonstrated appropriate absorption contrast and detector-limited spatial resolution. A special attention was paid to the 3D imaging of thick low-density foam materials to be used in design of laser fusion targets.
\end{abstract}

(c) 2010 Elsevier Ltd. All rights reserved.

\section{Introduction}

The soft X-ray microscopy (SXM) is well-known to benefit from its short working wavelength $(\lambda<10 \mathrm{~nm})$ and the inner electron shell resonance interaction with the materials. Since the first experiments in the early 80s at the BESSY synchrotron facility (Niemann et al., 1983; Schmahl et al., 1980, 1985), the "water window" microscopy in the spectral region $(2.4<\lambda<4.3 \mathrm{~nm}$ ) (Adam et al., 2005; Attwood, 2007; Johansson et al., 2004; Maser et al., 2000) has been supplemented with the soft X-ray photoelectron and NEXAFS spectromicroscopy (Hitchcock et al., 2008; Jacobsen et al., 2002), inner-cell soft X-ray tomography (Parkinson et al., 2008), coherent and incoherent imaging with laboratory X-ray sources (Adam et al., 2005; Brewer et al., 2007, 2008; Kim et al., 2006; Takman et al., 2007), etc. Despite of a number of impressive results obtained, there is rather a modest attitude to the current SXM technique, which seems to result from a remarkable progress in a number of alternative methods of high resolution imaging, such as confocal microscopy (Brakenhoff et al., 1985; Cox, 1984; Minsky, 1988;

\footnotetext{
* Corresponding author at: Lebedev Physical Institute, X-ray Optics Laboratory, 53 Leninsky Prospekt, 119991 Moscow, Russia. Tel.: +7 499783 3709; fax: +7 4991357880 .

E-mail address: iart@sci.lebedev.ru (I.A. Artyukov).
}

Pawley, 2006), low vacuum/environmental electron microscopy (Bogner et al., 2007; Danilatos, 1994), fluorescent UV microscopy (Arndt-Jovin et al., 1985; Bastiaens and Hell, 2004). In general, the real "stock value" of the SXM as an emerging research technique has still to be validated by the availability of the efficient and relatively inexpensive instrumentation and its yield of the exclusive information.

One of the known challenges in the modern biological microscopy is the investigation of thick organic samples (the thickness of many tens of microns) with a high spatial resolution and large depth of focus. Thus, we are witnessing impetuous progress in hard X-ray optical instrumentation for the high penetrating power wavelengths $\lambda \ll 1 \mathrm{~nm}$ (Thieme et al., 2003; Wang et al., 2001), with phase enhanced imaging techniques being applied to improve the imaging contrast while studying unstained biological samples.

On the other hand, there are a lot of mostly carbon-containing materials that are transparent enough to be studied by the methods of transmission SXM at the wavelengths of low absorption of the carbon - so called, "carbon window" (Artyukov et al., 2004). Fig. 1 shows this "carbon window" of transparency as the spectral region located just above the carbon K-edge, i.e. in the wavelength range $4.4<\lambda<5 \mathrm{~nm}$ (Henke et al., 1993). One can see also that a typical attenuation length of the soft X-rays exceeds 5 and $7 \mu \mathrm{m}$ inside carbon and paraffin, correspondingly. These values are the highest 


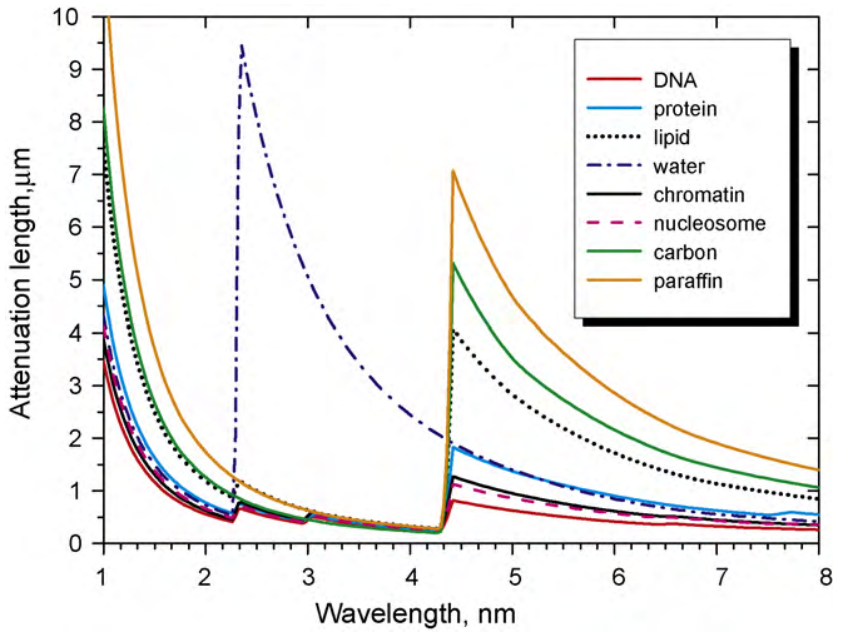

Fig. 1. The absorption of biological materials near the carbon K-edge.

over the entire soft X-ray/extreme ultraviolet (EUV) region at the wavelengths starting from $\lambda>1 \mathrm{~nm}$ (photon energy $E<1.2 \mathrm{keV}$ ).

While there is no visible contrast between water and proteins at the wavelength $\lambda \sim 4.5 \mathrm{~nm}$, one can see that an appropriate differential contrast occurs between other presented biological materials. The contrast pattern in the SXM images is known to result from the chemical composition of the organic materials containing different amount of highly absorptive elements (S, P, etc.), and in the case of "carbon window" this effect can be used for the recognition of most biological structures and reveal light traces of the heavy chemical elements inside "transparent" organic substances. In fact, it is the "carbon window" spectral region where a high contrast in the images of wet biological samples has been initially obtained at the BESSY before starting the first "water window" SXM experiments (Rudolph et al., 1984).

On the other hand, it has been proven that the importance of water environment in biological applications of the SXM is, to some degree, diluted by extremely destructive radiation doses absorbed by the cells and tissues during typical high resolution SXM studies. The cryogenic equipment for fixing the biological samples has become a "must have it" instrumentation in the "water window" SXM experiments. The only "water window" transparent solid substance - frozen water is rather a sophisticated fixing medium which requires the special efforts applied to avoid the inner-cellar membranes damage and other freezing-related artefacts.

The high transparency of hydrocarbons in the "carbon window" allows one to use a number of paraffin based standard fixing methods developed primarily for the visible light/UV microscopy and histological studies. To illustrate the efficiency of paraffin as a SXM fixing material, Fig. 2 shows simulations of the unstained transmission contrast patterns of $3-\mu \mathrm{m}$-thick slices calculated for typical biological materials embedded in paraffin. In the next sections we will present the correspondent experimental results to substantiate our concept of the high contrast biological SXM in the spectral region of the "carbon window".

The full "pro" list of the "carbon window" microscopy is:

- The lowest absorption of the most carbon-containing materials in the soft X-ray region enables:

- The absorption imaging of relatively thick samples with a high spatial resolution and large depth of focus; the 3D soft X-ray imaging and mapping are possible as well;

- The reduced radiation dose absorbed in relatively transparent organic structural elements. Note that the transmission of wet samples is still sufficient for their absorption studies in the "carbon window" SXM (the transmission of a $3 \mu \mathrm{m}$-thick water medium is $\sim 20 \%$ ).

- One can apply well-developed histological fixing and preserving techniques, e.g. those based on paraffin and formaldehyde compounds. In this case no cryogenic equipment would be needed for the SXM imaging, object handling and storing.

- Due to the low nitrogen absorption, the air is known to be much more transparent at the wavelengths of the "carbon window" $\lambda \sim 4.5 \mathrm{~nm}$ than at $\lambda \sim 2.4 \mathrm{~nm}$ resulting in less severe vacuum requirements in the SXM experiments (Rudolph et al., 1984). The free path length of the "carbon window" X-ray radiation in the ambient air is calculated to be about $1.46 \mathrm{~mm}$ in comparison with $0.59 \mathrm{~mm}$ at the wavelength $\lambda=2.4 \mathrm{~nm}$ (Henke et al., 1993).

- The carbon contamination of the optics (Chen et al., 2009; Shin et al., 2009) plays a minor role in the "carbon window" imaging experiments.

- The "carbon window" is a fully accessible operational region for the reflective $\mathrm{X}$-ray optics working at the normal incidence. The correspondent $\mathrm{Co} / \mathrm{C}$ multilayer mirrors provide the reflectivity of about 15\% (Artyukov et al., 2009). Reflective multilayer based two-mirror optical systems have been fabricated for both the "carbon window" and EUV spectral regions, and at the wavelength $\lambda \sim 13.5 \mathrm{~nm}$ they have achieved a spatial resolution of $22 \mathrm{~nm}$ (Naulleau et al., 2009). Evidently, the implementation of the similar high throughput and high resolution multilayer optics simplifies construction of a table-top soft X-ray microscope.

The following sections present our experiments that we carried out to test our approach to the "carbon window" SXM imaging on the basis of a relatively inexpensive and unsophisticated soft X-ray optical instrumentation and laboratory X-ray source.
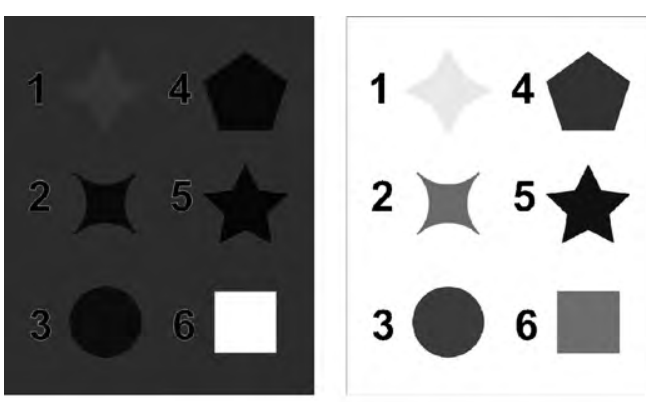

$\lambda=2.4 \mathrm{~nm}$

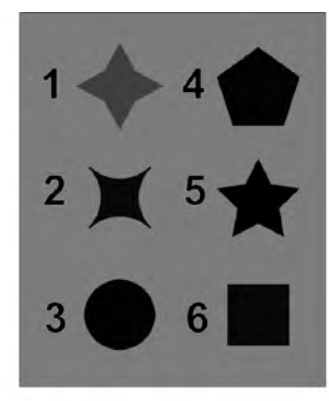

$\lambda=6.7 \mathrm{~nm}$

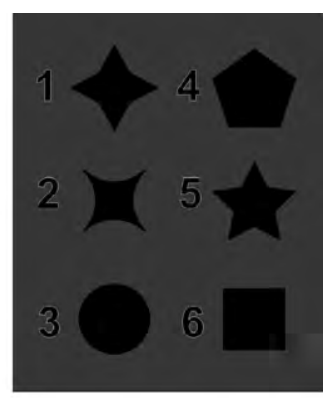

$\lambda=13.5 \mathrm{~nm}$

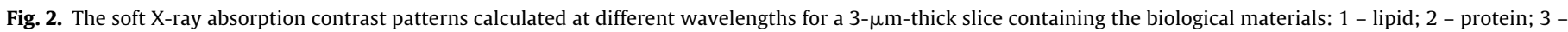
chromatin; 4 - nucleosome; 5 - DNA; 6 - water. The embedding material is paraffin. The brightness of the pattern at $\lambda=13.5 \mathrm{~nm}$ is increased in 100 times. 


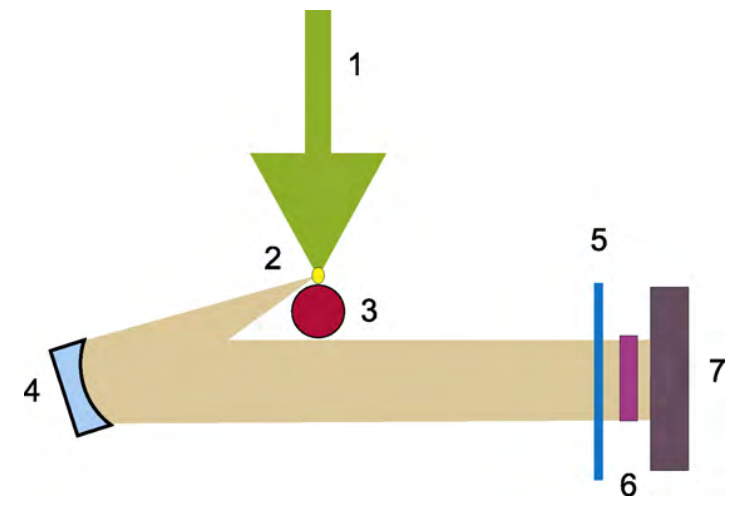

Fig. 3. The experimental set-up for the soft X-ray imaging at the wavelength of the "carbon window" $(\lambda \sim 4.5 \mathrm{~nm}): 1$ - the 2 nd harmonics radiation of the $N d-$ glass laser $(\lambda=0.53 \mu \mathrm{m}), 2$ - laser-produced plasma, 3 - bulk rhenium target, 4 spherical Co/C multilayer mirror, 5 - scandium thin filter, sample, 6 - sample under investigation on a mesh (or silicon nitride membrane) support, 7 - X-ray film UF-4. All components are isolated inside low vacuum chamber $\left(P \sim 10^{-2}\right.$ Torr $)$.

\section{Experimental set-up}

Our first experiments were aimed at demonstration of satisfactory absorption contrast of the "carbon window" images and the efficiency of the developed X-ray optical elements. The experimental set-up consists of the following components that were positioned inside the vacuum chamber with the low vacuum of $P=10^{-3}$ to $10^{-2}$ Torr (see Fig. 3).

The laser-produced plasma (LPP) was used as a compact soft Xray source. The plasma was generated using the FENIKS laser facility (Artioukov et al., 1995; Artsimovich et al., 1987) that could deliver up to $4 \mathrm{~J}$ of the pulse energy in the second harmonics $(\lambda=0.54 \mu \mathrm{m})$ of the $N d$ :glass laser onto a bulk rhenium target with the pulse duration of 1.2-2 ns and the focal spot size of 30-80 $\mu \mathrm{m}$. In practice, the typical laser pulse energy in the soft $\mathrm{X}$-ray imaging experiments was $0.3-1.2 \mathrm{~J}$.

The $\mathrm{Co} / \mathrm{C}$ multilayer coated spherical mirror (curvature radius $R=100 \mathrm{~mm}$, diameter $D=10 \mathrm{~mm}$ ), which served as a collimator and medium spectral resolution soft X-ray monochromator $(\Delta \lambda / \lambda \sim 1 / 200)$, was placed at the distance about $50 \mathrm{~mm}$ from the $\mathrm{X}$-ray source to produce a quasi-parallel soft X-ray beam at the wavelengths near $\lambda=4.5 \mathrm{~nm}$ (Artyukov et al., 2007, 2009). The visible and UV light of the laser plasma source was blocked with a set of two thin scandium polyimide-backed filters (Artioukov et al., 2003). The high normal incidence reflectivity of the $\mathrm{Co} / \mathrm{C}$ mirror and high transmission of the filters enabled soft X-ray imaging with one-nanosecond exposure, single laser shot and one joule pulse energy (so called "triple one mode").

The objects were fixed on a $500 \mu \mathrm{m}$ cell size copper mesh at the distance of about $40 \mathrm{~cm}$ from the mirror and in a close proximity to the surface of the film UF-4 (Datsko et al., 1987) used as a soft X-ray detector (the object-film gap was 100-200 $\mu \mathrm{m}$ ). The short working wavelength and low X-ray beam divergency brought the spatial resolution of our imaging experiments to that of the film. Thus, the spatial resolution of both the film UF- 4 and soft X-ray images was estimated to be $1-3 \mu \mathrm{m}$. The view field of $4 \mathrm{~mm}$ was determined by the open aperture of the filter used. The depth of focus was practically unlimited, which is typical for this type of Xray projection microradiography. To produce a series of four soft $\mathrm{X}$-ray images without breaking the vacuum condition we used a special film cassette.

The main difference of our experiments from the earlier works on X-ray contact (proximity) microscopy with a laser plasma source (see, foe example, Cefalas et al., 1998; Ford et al., 1991; Limongi et al., 2004; Michette et al., 1986; Milani et al., 2005) consists in

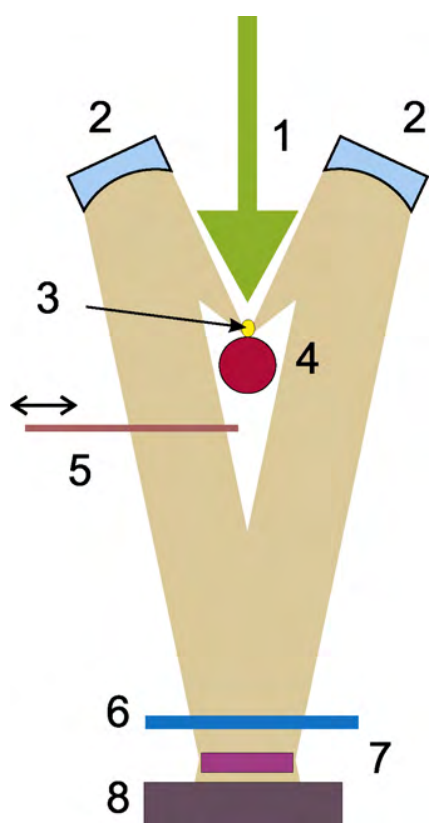

Fig. 4. The scheme for the soft X-ray stereo-microradiography: 1 - laser beam $(\lambda=0.53 \mu \mathrm{m}), 2$ - two spherical Co/C multilayer mirrors, 3 - laser-produced plasma 4 - rhenium target, 5 - left/right view shutter, 6 - scandium thin filter, 7 - sample, 6 - X-ray film UF-4. The parallax view angle is $7.8^{\circ}$.

introducing the normal incidence $X$-ray multilayer optics to reduce the working spectral bandwidth near the wavelength $\lambda \sim 4.5 \mathrm{~nm}$ to $\Delta \lambda \sim 0.02 \mathrm{~nm}$. As was mentioned above, multilayer mirrors can be used also in the high spatial resolution X-ray optical systems to put forward the "carbon window" microscopy from the schemes of the $1: 1$ projection microradiography to the multi-fold magnification microscopy (Artyukov et al., 2009; Dicicco et al., 1992; Murakami et al., 1993).

The micron-scale spatial resolution in combination with large thickness of many soft X-ray transparent samples made possible the effective 3D imaging, such as tomography scans or stereo views. Fig. 4 shows the modification of our experimental setup used for the production of soft X-ray stereo pairs - two identical spherical $\mathrm{X}$-ray multilayer mirrors were applied to provide the parallax view angle of $7.8^{\circ}$. The moving shutter enabled the sequential recording of two X-ray views of the same object at different angles.

\section{Examples of soft $X$-ray images}

The experimental study of contrast and transparency in the spectral region of the "carbon window" was carried out using the laser plasma based setups shown in Figs. 3 and 4. To produce absorption images of thick samples the composition of all objects under investigation was dominated by the carbon. Below are several examples of many images obtained in the experiments, which were chosen to represent the typical objects. As has been mentioned, all X-ray images were produced with nanosecond exposures.

\subsection{The wing edge of a grey fleshfly (Sarcophaga carnaria L.), untreated (Fig. 5)}

The fleshfly wing did not go under any special preparation process and was studied "as it is". ${ }^{1}$ The image demonstrates the

\footnotetext{
1 Of course, during the investigations in the vacuum conditions any object will be dehydrated.
} 


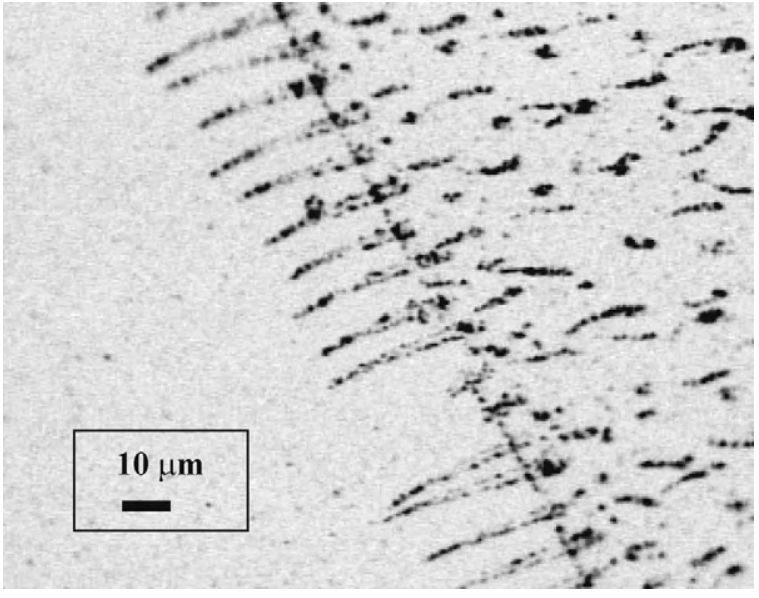

Fig. 5. The soft X-ray image of fleshfly wing $(\lambda \sim 4.5 \mathrm{~nm})$. Note the internal inhomogeneous structure of the wing hairs.

efficiency of the "carbon window" microscopy in application to the soft X-ray transmission studies of non-thinned and unstained biological objects. One can see a number of cavities and inhomogeneities inside the hair stems. These structures are hidden by the hair chitin absorption from a direct observation with the visible/UV, electron microscopy or "water window" SXM. On the other hand, it would be rather difficult to get such a clear view of the internal details using the methods of hard X-ray microscopy owing to a lower image contrast, which is inherent to the high energy radiation imaging. To reveal low contrast absorption structures one have to resort to more complicated experimental techniques based on the phase contrast, such as coherent diffraction (Clark et al., 2008; Miao et al., 1999) and diffraction enhanced (Chapman et al., 1997; Pagot et al., 2005) imaging.

The picture also displays the effect of film grain size limiting the spatial resolution.

\subsection{The edge of green bush-cricket (Terrigonia viridissima) wing, untreated (Fig. 6)}

Similar to the fleshfly wing this sample was studied without any special preparation or staining. Fig. 6B shows the visible light image of the same fragment, which, in contrast to the SXM picture (Fig. 6A), brings out noticeable distortion and diffraction effects near the sharp edges and focus depth limitation. The soft X-ray absorption pattern of the unstained sample proves to be different from that observed in the visible light.

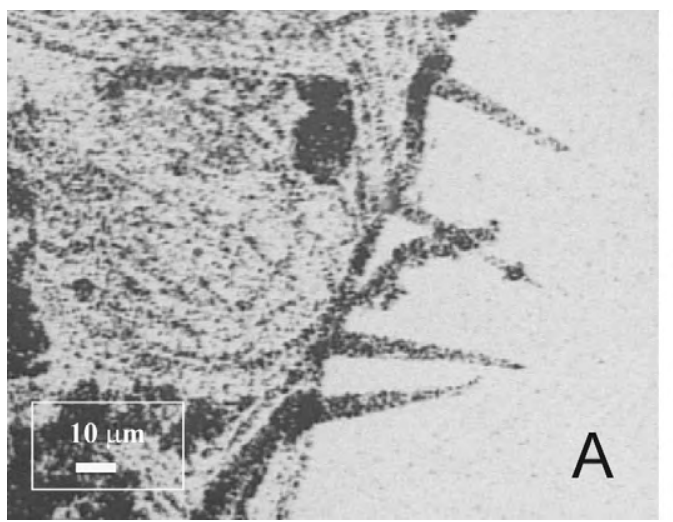

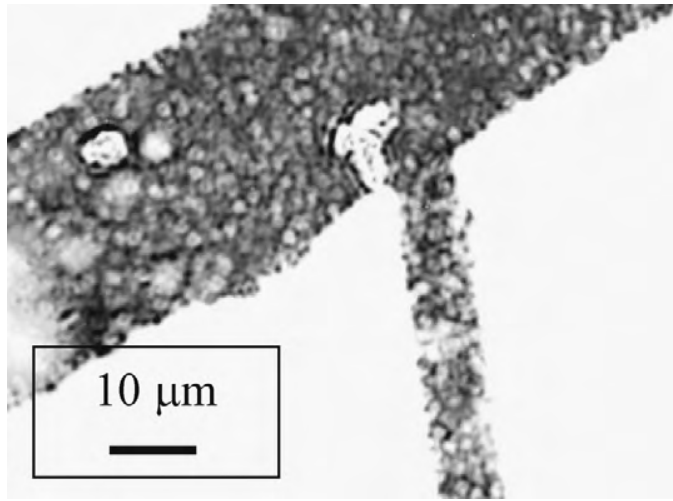

Fig. 7. The soft X-ray image of placental blood vessels $(\lambda \sim 4.5 \mathrm{~nm})$. The equal absorption in wide and narrow vessels indicates a tubular thin-wall form.

The estimated thickness of the sample under observation was 1-5 $\mu \mathrm{m}$.

\subsection{Placental blood vessels, preliminary desiccated (Fig. 7)}

Fig. 7 shows the internal structure of dried cotyledon blood vessels of human placenta. The object was dehydrated through the standard lyophilization process at a low temperature $\left(5-7^{\circ} \mathrm{C}\right)$. The observation of nearly equal X-ray transmission of large $(50-70 \mu \mathrm{m})$ and small $(\sim 10 \mu \mathrm{m})$ diameter vessel branches indicates their tubular structure.

\subsection{Blood vessel of embryo, sectioned and fixed in a paraffin bed (Fig. 8)}

This $10 \mu \mathrm{m}$-thick slice of human embryo (18 weeks) was prepared using the standard histological methods and substances: after lyophilization the dehydrated sample was fixed with paraffin compound and sectioned. The "carbon window" image of a $3 \mu \mathrm{m}-$ thick unstained slice depicts clearly the wall structure of the blood vessel. In comparison with the visible light microscopy (Fig. 8B) the soft X-ray image reveals numerous new details. Moreover, the paraffin filling the core center of the vessel looks to be even more transparent for the soft X-rays than for the visible light. The combination of the high contrast, high soft X-ray transmission and large depth of focus demonstrates the potential and penetrating power of the "carbon window" SXM.

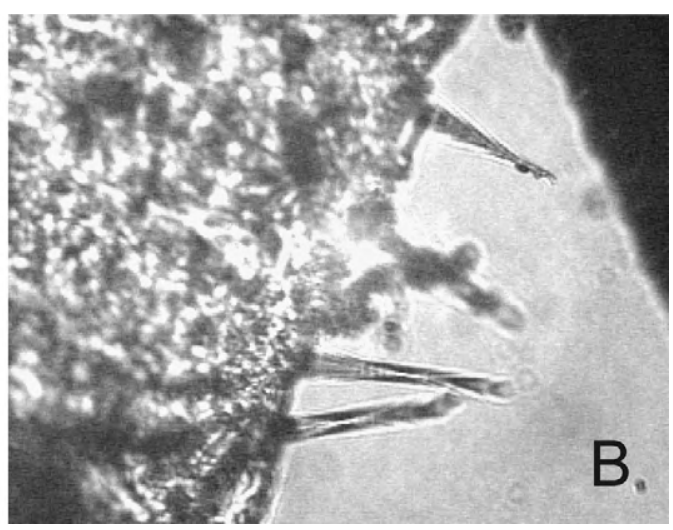

Fig. 6. Images of bush-cricket wing: (A) soft X-ray image at the wavelength $\lambda \sim 4.5 \mathrm{~nm}$; (B) the same fragment observed in the visible light. 

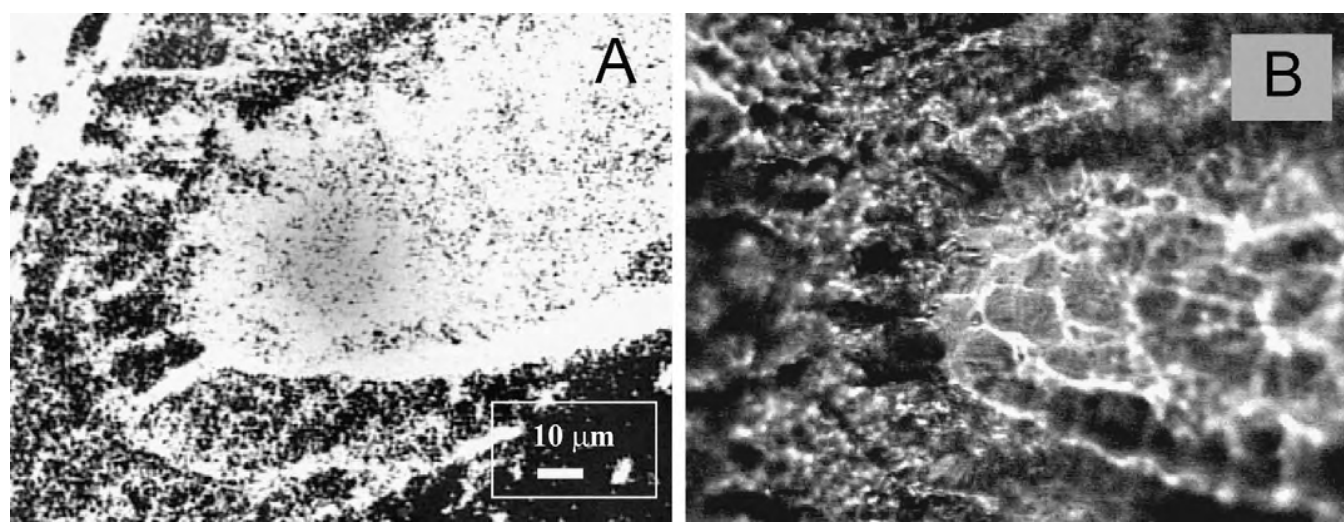

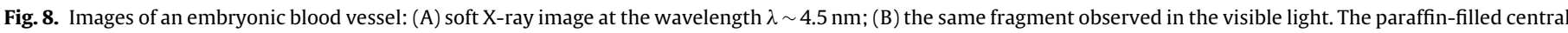
part of the vessel looks to be extremely transparent for the soft X-rays.

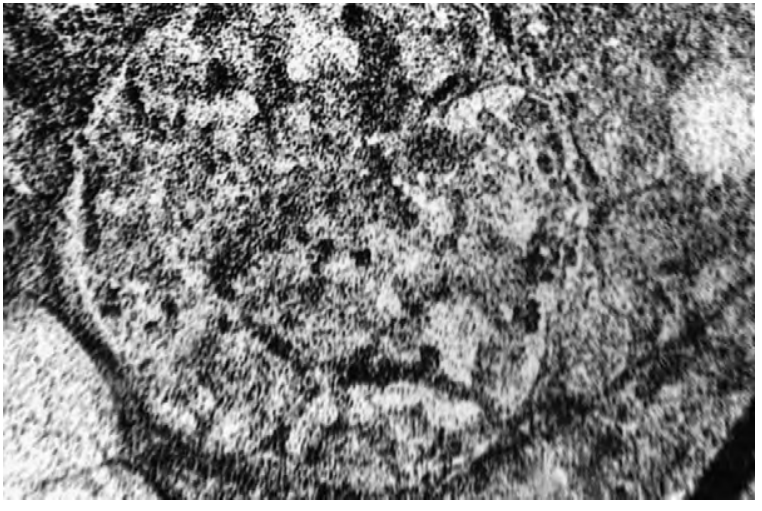

Fig. 9. The soft X-ray image of an unstained and unfixed multicell sample - pancreatic tissue $(\lambda \sim 4.5 \mathrm{~nm})$.

\subsection{Pancreatic tissue, sectioned and free of fixing compounds and water (Fig. 9)}

We prepared this sample to test the "carbon window" absorption contrast in the imaging of thick dehydrated soft tissues without application of coloring or contrasting techniques. Multicell tissue, which has been initially preserved in paraformaldehyde, was embedded in paraffin for the section cutting. After that the paraffin and other plasticizer substances were removed from the $10 \mu \mathrm{m}$-thick slices, and the wet slices were put atop thin silicon nitride membranes (thickness $100 \mathrm{~nm}$, working window size $2 \mathrm{~mm} \times 2 \mathrm{~mm}$ ) for the low temperature dehydration. Note that this type of the samples was the only one we investigated with the application of a thin silicon nitride membrane as a sample support instead of a supporting mesh.

Fig. 9 shows that the unstained SXM imaging can reveal a number of principal structural elements of the pancreas, such as pancreatic isles (insula Langerhansi); ducts (ductus pancreaticus) and blood capillaries.

\subsection{Polyacrylonitrile fibers (Fig. 10)}

Fig. 10 is the soft X-ray image of polyacrylonitrile (PAN) fibers (Sedghi et al., 2008). This type of the carbon materials has been used widely as a basic component of various composite materials with the improved mechanical properties. The picture clearly reveals internal inhomogeneities, substructures and cracks inside the $10 \mu \mathrm{m}$-diameter fibers. The film grain size was proven to limit the spatial resolution and information about the disk-like skeleton structure of the PAN-fibers. The recently developed X-ray

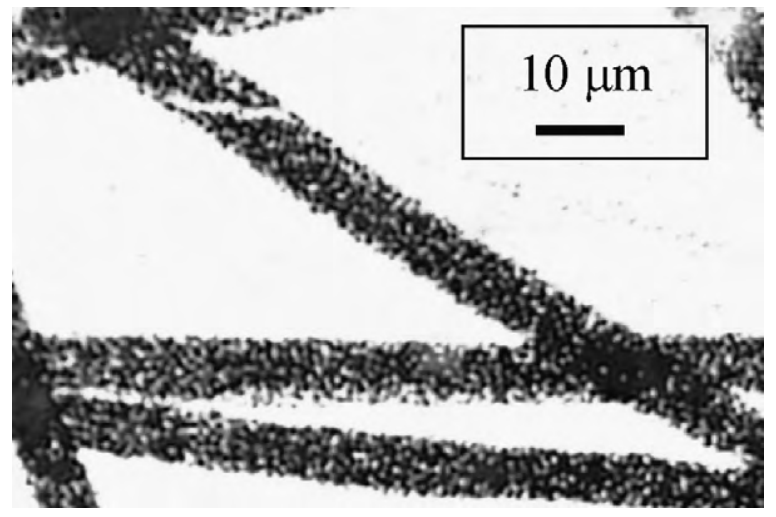

Fig. 10. The soft X-ray image of polyacrylonitrile (PAN) fibers $(\lambda \sim 4.5 \mathrm{~nm})$. The limited spatial resolution (film grains) hinders from viewing the details of the internal structure.

Schwarzschild objective (Artyukov et al., 2009) can significantly improve the resolution to turn this SXM technique to an effective tool for introscopic investigations of graphite fibers.

\subsection{Low-density foams and TAC materials (Fig. 11)}

The low-density triacetate of cellulose (TAC) is considered as an important material for the construction of a new generation of laser fusion targets (Limpouch et al., 2004; Nagai et al., 2005). The extremely low density $\left(\rho=1.7-10 \mathrm{mg} / \mathrm{cm}^{3}\right)$ and low radiation damage threshold make these materials difficult to observe using the transmission electron microscopy (TEM) or scanning electron microscopy (SEM). The high transparency of organic materials in the "carbon window" reduces the radiation absorption dose and increases the practical depth of observation up to several millimeters.

In comparison with the visible light microscopy the SXM technique is capable of producing the images with a significantly larger depth of focus (which is practically unlimited in the one-to-one projection imaging). The strong soft $\mathrm{X}$-ray absorption of the metals enables to detect them in light trace concentrations, to map their 3D distribution inside a bulk TAC material and to check the physical uniformity, which is critical for its applications as a laser fusion target (see Fig. 11A).

Additional prospects in the SXM based studies of TAC materials arise with the introduction of 3D view. In general, the high ratio of the object thickness to spatial resolution (up to $10^{3}$ ), that is specific for the "carbon window" imaging, establishes a solid ground for the production of soft X-ray 3D images. Besides that, the large thickness 

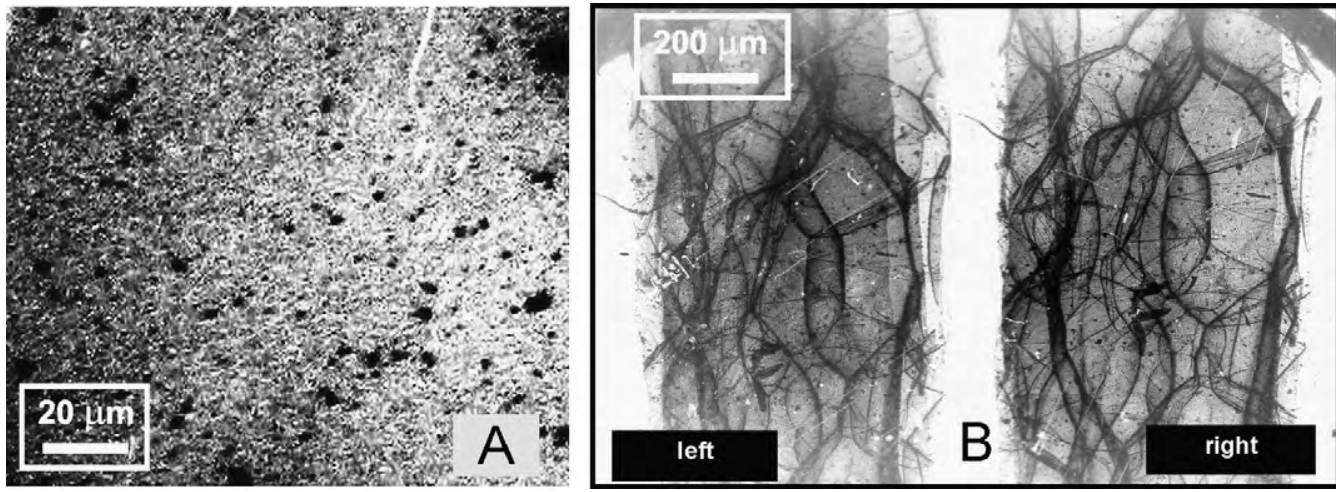

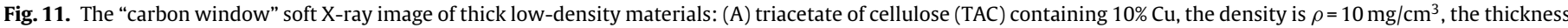
is $0.3 \mathrm{~mm}$; (B) stereoimage of blank organic foam with the thickness of $0.8 \mathrm{~mm}$.

of transparent objects enables soft X-ray survey of large volume organic samples.

Our first 3D view demonstration experiments consisted in production of the soft X-ray stereo pairs and anaglyphs of low-density foam materials with the thickness up to $2 \mathrm{~mm}$. The obtained "carbon window" images demonstrate a clear and contrast view of the spatial distribution of volumetric structure elements (see Fig. 11B).

\section{Conclusions}

The normal incidence X-ray multilayer optics has been proven to create new opportunities for the SXM technique in the spectral region of the "carbon window". We demonstrated a satisfactory Xray contrast in plenty of $2 \mathrm{D}$ and $3 \mathrm{D}$ images without any contrasting or staining additives. The spatial resolution was limited by the $\mathrm{X}$ ray film grain size and can be improved by a factor of 20-50 with the help of the recently developed X-ray Schwarzschild objective down to the level of $\sim 100 \mathrm{~nm}$. Another direction of our future work is related to the implementation of thin membrane cells for studying wet samples under the vacuum conditions.

The "carbon window" microscopy based on the multilayer optics and laboratory X-ray source appears to be an attractive and effective technique for the absorption study of thick organic materials, which is supplementary to the "water window" X-ray imaging. Of course, its lot in life should be supported by an active interest of interscience users and practical SXM applications.

\section{Acknowledgements}

The authors are thankful to Dr. Nikolai Melnik, Dr. Yu.A. Merkuliev and Dr. Alexander Shevelko (P.N. Lebedev Physical Institute of RAS, Moscow) for their kind help and technical support of this work. The project was funded in part by the US Civilian Research \& Development Foundation, \# RP2-2845-MO-02 and the Russian Foundation for Basic Research, \#\# 07-02-01244-a, 07-0201148-a, 10-02-00991-a and 08-08-00080-a.

\section{References}

Adam, J.F., Moy, J.P., Susini, J., 2005. Table-top water window transmission X-ray microscopy: review of the key issues, and conceptual design of an instrument for biology. Rev. Sci. Instrum. 76, 091301.

Arndt-Jovin, D., Robert-Nicoud, M., Kaufman, S., Jovin, T., 1985. Fluorescence digital imaging microscopy in cell biology. Science 230, 247-256.

Artioukov, I.A., Kasyanov, Y.S., Kopylets, I.A., Pershin, Y.P., Romanova, S.A., 2003. Scandium/carbon filters for soft $X$ rays. Rev. Sci. Instrum. 74, 4964-4966.

Artioukov, I.A., Vinogradov, A.V., Asadchikov, V.E., Kasyanov, Y.S., Serov, R.V., Fedorenko, A.I., Kondratenko, V.V., Yulin, S.A., 1995. Schwarzschild soft-X-ray microscope for imaging of nonradiating objects. Opt. Lett. 20, 2451-2453.
Artsimovich, V.L., Gaponov, S.V., Kas'yanov, Y.S., Luskin, B.M., Salashchenko, N.N., Sobel'man, I.I., Shevel'ko, A.P., 1987. Formation of directed intense vacuum ultraviolet radiation from a laser plasma. JETP Lett. 46, 391-394.

Artyukov, I., Bugayev, Y., Devizenko, O., Gullikson, E., Kondratenko, V., Vinogradov, A., 2009. X-ray Schwarzschild objective for the carbon window (lambda $\sim 4.5 \mathrm{~nm}$ ). Opt. Lett. 34, 2930-2932.

Artyukov, I.A., Bugayev, Y.A., Devizenko, O.Y., Feshchenko, R.M., Hatano, T. Kasyanov, Y.S., Kondratenko, V.V., Uspenski, Y.A., Vinogradov, A.V., 2007. Advances in short-wavelength X-ray multilayer optics: toward high-throughput multimirror systems for the wavelengths $<10 \mathrm{~nm}$, Soft X-ray Lasers and Applications VII. In: Proceedings of the SPIE, San Diego, CA, USA, p. 67020V.

Artyukov, I.A., Vinogradov, A.V., Kas'yanov, Y.S., Savel'ev, S.V., 2004. X-ray microscopy in the carbon window region. Quant. Electron. 34, 691-692.

Attwood, D.T., 2007. Soft X-rays and Extreme Ultraviolet Radiation: Principles and Applications. Cambridge University Press, Cambridge, New York.

Bastiaens, P.I.H., Hell, S.W., 2004. Light microscopy on the move. J. Struct. Biol. 147, $1-2$.

Bogner, A., Jouneau, P.H., Thollet, G., Basset, D., Gauthier, C., 2007. A history of scanning electron microscopy developments: towards "wet-STEM" imaging. Micron 38, 390-401.

Brakenhoff, G.J., Vandervoort, H.T.M., Vanspronsen, E.A., Linnemans, W.A.M., Nanninga, N., 1985. 3-Dimensional chromatin distribution in neuro-blastoma nuclei shown by confocal scanning laser microscopy. Nature 317, 748-749.

Brewer, C.A., Brizuela, F., Martz, D., Vaschenko, G., Marconi, M.C., Chao, W., Anderson, E.H., Attwood, J.D.T., Vinogradov, A.V., Artioukov, I.A., Pershyn, Y.P., Kondratenko, V.V., Rocca, J.J., Menoni, C.S., 2007. High spatial resolution full-field microscopy using a desktop-size soft X-ray laser, Soft X-ray Lasers and Applications VII. In: Proceedings of the SPIE, San Diego, CA, USA, pp. 67020M-67027M

Brewer, C.A., Brizuela, F., Wachulak, P., Martz, D.H., Chao, W., Anderson, E.H. Attwood, D.T., Vinogradov, A.V., Artyukov, I.A., Ponomareko, A.G., Kondratenko, V.V., Marconi, M.C., Rocca, J.J., Menonil, C.S., 2008. Single-shot extreme ultraviolet laser imaging of nanostructures with wavelength resolution. Opt. Lett. 33, $518-520$.

Cefalas, A.C., Argitis, P., Kollia, Z., Sarantopoulou, E., Ford, T.W., Stead, A.D., Marranca, A., Danson, C.N., Knott, J., Neely, D., 1998. Laser plasma X-ray contact microscopy of living specimens using a chemically amplified epoxy resist. Appl. Phys. Lett. $72,3258-3260$.

Chapman, D., Thomlinson, W., Johnston, R.E., Washburn, D., Pisano, E., Gmur, N., Zhong, Z., Menk, R., Arfelli, F., Sayers, D., 1997. Diffraction enhanced X-ray imaging. Phys. Med. Biol. 42, 2015-2025.

Chen, J., Louis, E., Lee, C.J., Wormeester, H., Kunze, R., Schmidt, H., Schneider, D. Moors, R., van Schaik, W., Lubomska, M., Bijkerk, F., 2009. Detection and characterization of carbon contamination on EUV multilayer mirrors. Opt. Express 17 16969-16979.

Clark, J.N., Williams, G.J., Quiney, H.M., Whitehead, L., de Jonge, M.D., Hanssen, E., Altissimo, M., Nugent, K.A., Peele, A.G., 2008. Quantitative phase measurement in coherent diffraction imaging. Opt. Express 16, 3342-3348.

Cox, I.J., 1984. Scanning optical fluorescence microscopy. J. Microsc.-Oxford 133 $149-154$.

Danilatos, G.D., 1994. Environmental scanning electron-microscopy and microanalysis. Mikrochim. Acta 114, 143-155.

Datsko, I.M., Slabkovskaya, M.A., Sokolov, A.S., Uvarova, N.V., Sheromov, M.A., 1987. Absolute calibration of X-ray films using synchrotron radiation. Nucl. Instrum. Methods Phys. Res. Sect. A: Accelerators, Spectrom. Detect. Assoc. Equip. 261, 327-329.

Dicicco, D.S., Kim, D., Rosser, R., Suckewer, S., 1992. 1st stage in the development of a soft-X-ray reflection imaging microscope in the Schwarzschild configuration using a soft-X-ray laser at $18.2 \mathrm{~nm}$. Opt. Lett. 17, 157-159.

Ford, T.W., Stead, A.D., Cotton, R.A., 1991. Soft X-ray contact microscopy of biological materials. Electron. Microsc. Rev. 4, 269-292.

Henke, B.L., Gullikson, E.M., Davis, J.C., 1993. X-ray interactions-photoabsorption, scattering, transmission, and reflection at $E=50-30,000 \mathrm{Ev}, Z=1-92$. Atom. Data Nucl. Data 54, 181-342. 
Hitchcock, A.P., Dynes, J.J., Johansson, G., Wang, J., Botton, G., 2008. Comparison of NEXAFS microscopy and TEM-EELS for studies of soft matter. Micron 39, 311-319.

Jacobsen, C., Beetz, T., Feser, M., Osanna, A., Stein, A., Wirick, S., 2002. Spectromicroscopy of biological and environmental systems at Stony Brook: instrumentation and analysis. Surf. Rev. Lett. 9, 185-191.

Johansson, G.A., Khanna, S.M., Nair, A., Mannström, P., Denbeaux, G., Ulfendahl, M., 2004. Exploring the use of soft X-ray microscopy for imaging subcellular structures of the inner ear. J. Microsc. 215, 203-212.

Kim, K.W., Kwon, Y., Nam, K.Y., Lim, J.H., Kim, K.G., Chon, K.S., Kim, B.H., Kim, D.E., Kim, J., Ahn, B.N., Shin, H.J., Rah, S., Kim, K.H., Chae, J.S., Gweon, D.G., Kang, D.W., Kang, S.H., Min, J.Y., Choi, K.S., Yoon, S.E., Kim, E.N., Namba, Y., Yoon, K.H., 2006. Compact soft X-ray transmission microscopy with sub-50 $\mathrm{nm}$ spatial resolution. Phys. Med. Biol. 51, N99-N107.

Limongi, T., Palladino, L., Tomassetti, G., Reale, L., Cesare, P., Flora, F., Aimola, P., Ragnelli, A.M., 2004. Comparative analysis of isolated cellular organelles by means of soft X-ray contact microscopy with laser-plasma source and transmission electron microscopy. J. Microsc. 214, 43-50.

Limpouch, J., Demchenko, N.N., Gus'kov, S.Y., Kalal, M., Kasperczuk, A., Kondrashov, V.N., Krousky, E., Masek, K., Pisarczyk, P., Pisarczyk, T., Rozanov, V.B., 2004. Laser interactions with plastic foam-metallic foil layered targets. Plasma Phys. Control. Fusion 46, 1831-1841.

Maser, O., Wang, J., Kirz, S., Winn, T., 2000. Soft X-ray microscopy with a cryo scanning transmission X-ray microscope: I. Instrumentation, imaging and spectroscopy. J. Microsc. 197, 68-79.

Miao, J., Charalambous, P., Kirz, J., Sayre, D., 1999. Extending the methodology of $\mathrm{X}$-ray crystallography to allow imaging of micrometre-sized non-crystalline specimens. Nature 400, 342-344

Michette, A.G., Cheng, P.C., Eason, R.W., Feder, R., Oneill, F., Owadano, Y., Rosser, R.J., Rumsby, P., Shaw, M.J., 1986. Soft-X-ray contact microscopy using laser plasma sources. J. Phys. D: Appl. Phys. 19, 363-372.

Milani, M., Drobne, D., Tatti, F., Batani, D., Poletti, G., Orsini, F., Zullini, A., Zrimec, A. 2005. Read-out of soft X-ray contact microscopy microradiographs by focused ion beam/scanning electron microscope. Scanning 27, 249-253.

Minsky, M., 1988. Memoir on inventing the confocal scanning microscope. Scanning $10,128-138$.

Murakami, K., Oshino, T., Nakamura, H., Ohtani, M., Nagata, H., 1993. Schwarzschild microscope for carbon $\mathrm{K} \alpha$ radiation. Appl. Opt. 32, 7057-7061.

Nagai, K., Azechi, H., Ito, F., Iwamoto, A., Izawa, Y., Johzaki, T., Kodama, R., Mima, K., Mito, T., Nakai, M., Nemoto, N., Norimatsu, T., Ono, Y., Shigemori, K., Shiraga, H., Tanaka, K.A., 2005. Foam materials for cryogenic targets of fast ignition realization experiment (FIREX). Nucl. Fusion 45, 1277-1283.
Naulleau, P.P., Anderson, C.N., Chiu, J., Denham, P., George, S., Goldberg, K.A., Goldstein, M., Hoef, B., Hudyma, R., Jones, G., Koh, C., Fontaine, B.L., Ma, A. Montgomery, W., Niakoula, D., Park, J.-o., Wallow, T., Wurm, S., 2009. 22-nm Half-pitch extreme ultraviolet node development at the SEMATECH Berkeley microfield exposure tool. Microelectron. Eng. 86, 448-455.

Niemann, B., Rudolph, D., Schmahl, G., 1983. The Göttingen X-ray microscopes. Nucl. Instrum. Methods Phys. Res. 208, 367-371.

Pagot, E., Fiedler, S., Cloetens, P., Bravin, A., Coan, P., Fezzaa, K., Baruchel, J., Hartwig, J. 2005. Quantitative comparison between two phase contrast techniques: diffraction enhanced imaging and phase propagation imaging. Phys. Med. Biol. 50, 709-724.

Parkinson, D.Y., McDermott, G., Etkin, L.D., Le Gros, M.A., Larabell, C.A., 2008. Quantitative 3-D imaging of eukaryotic cells using soft X-ray tomography. J. Struct. Biol. 162, 380-386.

Pawley, J.B., 2006. Handbook of Biological Confocal Microscopy, 3rd ed. Springer New York, NY.

Rudolph, D., Niemann, B., Schmahl, G., Christ, O., 1984. The Göttintgen X-ray microscope and X-ray microscopy experiments at the BESSY storage ring. In: Schmahl, G., Rudolph, D. (Eds.), X-ray Microscopy: Proceedings of the International Symposium. Göttingen, Fed. Rep. of Germany, September 14-16, 1983. Springer-Verlag, Berlin; New York, pp. 192-202.

Schmahl, G., Niemann, B., Rudolph, D., Guttmann, P., Sarafis, V., 1985. X-ray microscopy: experimental results with the Gottingen X-ray microscope at the electron storage ring BESSY in Berlin. J. Microsc. 138, 279-284.

Schmahl, G., Rudolph, D., Niemann, B., Christ, O., 1980. Zone-plate X-ray microscopy. Quart. Rev. Biophys. 13, 297-315.

Sedghi, A., Farsani, R.E., Shokuhfar, A., 2008. The effect of commercial polyacrylonitrile fibers characterizations on the produced carbon fibers properties. J. Mater. Process. Technol. 198, 60-67.

Shin, H., Sporre, J.R., Raju, R., Ruzic, D.N., 2009. Reflectivity degradation of grazing-incident EUV mirrors by EUV exposure and carbon contamination. Microelectron. Eng. 86, 99-105.

Takman, P.A.C., Stollberg, H., Johansson, G.A., Holmberg, A., Lindblom, M., Hertz, H.M., 2007. High-resolution compact X-ray microscopy. J. Microsc.-Oxford 226 $175-181$.

Thieme, J., Schneider, G., Knochel, C., 2003. X-ray tomography of a microhabitat of bacteria and other soil colloids with sub-100 $\mathrm{nm}$ resolution. Micron 34 , 339-344.

Wang, Y., Carlo, F.D., Mancini, D.C., McNulty, I., Tieman, B., Bresnahan, J., Foster, I, Insley, J., Lane, P., Laszewski, G.v., Kesselman, C., Su, M.-H., Thiebaux, M., 2001. A high-throughput X-ray microtomography system at the Advanced Photon Source. Rev. Sci. Instrum. 72, 2062-2068. 